\title{
Guías de la red EQUATOR y sus listas de verificación: herramientas para publicar con calidad
}

\section{EQUATOR Network Guidelines and their checklists: Tools for quality publishing}

\section{Luis A. Gorordo-Delsol1,2*}

${ }^{1}$ Unidad de Cuidados Intensivos de Adultos, Hospital Juárez de México; Editor Jefe, Revista de Educación en Investigación en Emergencias, Sociedad Mexicana de Medicina de Emergencia A.C. Ciudad de México, México

La red EQUATOR (Enhancing the QUAlity and Transparency Of health Research) es una organización que promueve la calidad y transparencia en la investigación en salud por medio de estandarización de los elementos básicos, es decir, mínimos indispensable, de diferentes tipos de manuscrito ${ }^{1,2}$.

Las listas de verificación cubren 12 tipos de estudios y cada una de estas listas tiene versiones específicas para diversas metodologías u objetivos de las publicaciones (Tabla 1), por esto, cada vez más revistas sugieren - incluso exigen - utilizar estas listas de cotejo para estructurar los trabajos que se someten a revisión, y que deben ser consideradas seriamente cuando se inicie un proyecto de investigación ${ }^{3}$ y cuando se decida publicar los resultados; ; simultáneamente, la red EQUATOR ofrece una serie de herramientas ${ }^{5}$ a los

Tabla 1. Guías de la red EQUATOR (Enhancing the QUAlity and Transparency Of health Research)

\begin{tabular}{|l|l|}
\hline Tipo de estudio & Guías de reporte \\
\hline Estudios controlados aleatorizados & CONSORT (CONsolidated Standards of Reporting Trials) \\
\hline Estudios observacionales & STROBE (Strengthening the Reporting of Observational Studies in Epidemiology) \\
\hline Protocolos de estudio & SPIRIT (Standard Protocol Items: Recommendations for Interventional Trials) \\
\hline Protocolos de revisiones sistemáticas & PRISMA-P (PRISMA for protocols) \\
\hline Revisiones sistemáticas & PRISMA (Preferred Reporting Items for Systematic Reviews and Meta-Analyses) \\
\hline Estudios de diagnóstico y/o pronóstico & STARD (STAndards for the Reporting of Diagnostic accuracy studies) \\
\hline Reportes de caso & CARE (Case Reports) \\
\hline Guías de práctica clínica & AGREE (Appraisal of Guidelines for Research and Evaluation) \\
\hline Investigación cualitativa & SROR (Standards for Reporting Qualitative Research) \\
\hline Estudios preclínicos en animales & ARRIVE (Animal Research: Reporting of In Vivo Experiments) \\
\hline Estudios de mejoría de la calidad & SQUIRE (Standards for QUality Improvement Reporting Excellence) \\
\hline Estudios de evaluación económica & CHEERS (Consolidated Health Economic Evaluation Reporting Standards) \\
\hline
\end{tabular}

\section{Correspondencia:}

*Luis A. Gorordo-Delsol

E-mail: luis.gorordodelsol@icloud.com
Disponible en internet: 25-11-2021 Rev Educ Investig Emer. 2021;3(4):187-188 www.medicinadeemergencias.com

2604-6520 (C) 2021 Sociedad Mexicana de Medicina de Emergencias, AC. Publicado por Permanyer México SA de CV. Este es un artículo open access bajo la licencia CC BY-NC-ND (http://creativecommons.org/licenses/by-nc-nd/4.0/). 
revisores para un análisis objetivo y puntual de los elementos de un buen manuscrito, de tal forma que no solo homologan como se debe escribir, sino como se debe evaluar.

Finalmente, considero muy importante que los lectores - clínicos, investigadores, docentes y estudiantes - conozcan estas herramientas y los elementos que incluye cada guía de publicación para poder realizar un análisis crítico de los artículos publicados ${ }^{6,7}$ durante la planeación de un protocolo, la toma de decisiones clínicas y los ejercicios académicos propios de la medicina.

Por todo lo anterior, la Revista de Educación e Investigación en Emergencias emprenderá un proceso de aplicación de estas guías en las "Instrucciones para Autores" que incluye: capacitación y difusión de estas guías, ajuste de las instrucciones, con lo que se dará prioridad a los artículos que se ciñan a estos lineamientos internacionalmente aceptados, para así lograr la publicación de artículos de mayor calidad científica.

\section{Financiamiento}

No se recibió financiamiento para este trabajo.

\section{Conflicto de intereses}

El autor declara no tener conflictos de intereses.

\section{Bibliografía}

1. EQUATOR Network Running title Enhancing the Quality and Transparency of Health Research; 2021 [acceso: 1 de octubre de 2021]. Disponible en: https://www.equator-network.org

2. Gorordo-Delsol LA. ¿Por qué publicar reportes de casos? Rev Educ Investig Emer. 2021;3(3):119.

3. Albavera-Hernández C, Rodríguez-Rojas S, Gardía-Orihuela I, Baltazar-Moráles S, Meza-Rodríguez AB. ¿Cómo realizar un proyecto de investigación? Rev Educ Investig Emer. 2021;3(3):162-73.

4. López-Romero G, Castillo-Pérez JJ, Montiel-Jarquin AJ, Carrillo-Montes MA Ramírez-Sánchez SC, Villatoro-Martínez A. Cómo publicar en medicina de urgencia. Rev Educ Investig Emer. 2021;3(1):36-41.

5. EQUATOR Network [sede web]. Oxford, Reino Unido: Enhancing the Quality and Transparency of Health Research: Peer reviewing research; 2021 [acceso: 1 de octubre de 2021]. Disponible en: https://www.equator-network.org/toolkits/peer-reviewing-research

6. Faggion Jr CM. EQUATOR reporting guidelines should also be used by clinicians. J Clin Epidemiol. 2020;117:149-50.

7. Rivas-Ruiz R, Roy-García IA, Pérez-Rodríguez M, Moreno-Noguez M, Flores-Pulido A, Jiménez-Martínez MC, et al. \#Publica \#IniciativaM: publica mejor. Rev Educ Investig Emer. 2021;3(2):100-4. 